\title{
Concepts of the Last Eukaryotic Common Ancestor
}

\section{Authors and affiliations}

$\S^{\star}$ Maureen A. O'Malley, LaBRI, University of Bordeaux; HPS, University of Sydney, Sydney NSW, Australia, 2006

§Michelle M. Leger, Institute of Evolutionary Biology, UPF-CSIC, Barcelona, Pg. Marítim de la Barceloneta 39-47, Barcelona, Spain, 08003

Jeremy G. Wideman, Wissenschaftskolleg zu Berlin, Wallotstrasse 19, 14193, Berlin, Germany. Biochemistry and Molecular Biology, Dalhousie University, 5850 College Street, Halifax, Nova Scotia, Canada, B3H4R2

*Iñaki Ruiz-Trillo, Institute of Evolutionary Biology, Barcelona, UPF-CSIC, Barcelona, Pg. Marítim de la Barceloneta 39-47, Barcelona, Spain, 08003; \& Departament de Genètica, Microbiologia i Estadística, Facultat de Biologia, Institut de Recerca de la Biodiversitat (IRBio), Universitat de Barcelona (UB), Barcelona 08028, Catalonia, Spain; \& ICREA, Pg. Lluís Companys 23, 08010 Barcelona, Catalonia, Spain

*Correspondence to MAO: maureen.omalley@sydney.edu.au and IR-T: inaki.ruiz@ibe.upfcsic.es

$\S$ Co-first authors

\begin{abstract}
Insight into the Last Eukaryotic Common Ancestor (LECA) is central to any phylogeny-based reconstruction of early eukaryotic evolution. Increasing amounts of data enable such reconstructions, without necessarily sharpening our view of what LECA actually was. We consider four possible concepts of LECA: as an abstract phylogenetic state, a single cell, a population, and a consortium of organisms. We argue that the view most realistically underlying work in the field is that of LECA as a population. Drawing on recent findings of genomically heterogeneous populations in eukaryotes ('pangenomes'), we examine the evolutionary implications of a pangenomic LECA population. For instance, how does this concept affect standard expectations about the ecology, geography, fitness, and diversification of LECA? Does it affect evolutionary interpretations of LECA's cellular functions? Finally, we examine whether this novel pangenomic concept of LECA has implications for phylogenetic reconstructions of early eukaryote evolution. Our aim is to add to the conceptual toolkit for developing theories of LECA and interpreting genomic datasets.
\end{abstract}

\section{Keywords}

Last Eukaryotic Common Ancestor, LECA, eukaryote origins, eukaryote evolution, eukaryote phylogeny, eukaryotic pangenomes 


\section{Introduction and motivation}

Phylogeny-based reconstructions of ancestral states are central to understanding evolutionary history. To facilitate interpretations of these reconstructions and the inferred evolutionary histories of ancestral organisms, researchers use concepts such as the Last Universal Common Ancestor (LUCA), the Last Eukaryotic Common Ancestor (LECA), or the Last Metazoan Common Ancestor. However, these concepts may mean different things to different researchers, and their implications may not have been thoroughly examined. Here, we focus on LECA, which lies at the heart of many recent phylogenetic and evolutionary disputes.

Although the main issue in these debates is the evolutionary order and importance of specific inferred features of LECA, we have a different target. Our goal is to identify the actual and potential concepts of LECA that inform reconstructions of its features. We then analyse the evolutionary implications of these views. The full range of LECA concepts is rarely discussed explicitly, and unstated assumptions may influence how researchers interpret the early evolution of eukaryotes. To achieve our aim of providing a conceptual resource for evolutionary reconstruction, we first spell out the four possible concepts of LECA: as a hypothetical reconstruction, an individual cell, a population, and a consortium of different lineages. We clarify each of these conceptual variants, explore their implications, and consider more closely a novel pangenomic view of LECA. We suggest that the pangenomic view of LECA may affect the evolutionary questions to be addressed by ancestral eukaryotic reconstructions.

\section{What is LECA?}

LECA is, by definition, the ancestral state that gave rise to all extant eukaryotes. An important initial clarification is that LECA is distinct from the First Eukaryotic Common Ancestor (FECA) ${ }^{1}$ (Figure 1). FECA is commonly represented as the first descendant (on the eukaryotic branch) of the last common ancestral node of eukaryotes and an 'archaeal' lineage ${ }^{1-3}$ (the latter now believed to be related to the Asgard group ${ }^{4}$ ). In other words, FECA is the oldest ancestor of eukaryotes that is not also an ancestor of an extant archaeal lineage (note: this concept is agnostic about the acquisition of the mitochondrion). FECA is thus the ancestor of all eukaryotes that ever existed, whether extant or extinct. By contrast, LECA is the ancestor only of extant eukaryotes (all the known ones), plus extinct post-LECA lineages. This distinction means that LECA post-dates any early eukaryotic or 'proto-eukaryotic' lineages that have since become extinct. LECA was not, therefore, the first biological entity to possess recognizably eukaryotic features ${ }^{1,5}$. But even when LECA is distinguished from FECA, the bare definition of being ancestral to all extant eukaryotes is not exhaustive of how this major turning point in evolutionary history can be conceptualized.

\section{Figure 1. A continuum of LECA conceptions.}

A graphic depiction of how evolutionary researchers can conceptualize LECA. State $A$ is the overarching hypothetical state, and States B, C, D, and E represent all possible biological states. $E$ is seldom mentioned in the literature; $D$ is our novel pangenomic populational concept of LECA. 
A: a hypothetical ancestral reconstruction; $\mathrm{B}$ : a single cell; C: a genetically homogeneous population; D: a genomically heterogeneous (i.e., pangenomic) population; E: a consortium (including prokaryotic symbionts).

Hypothetical ancestral states

A

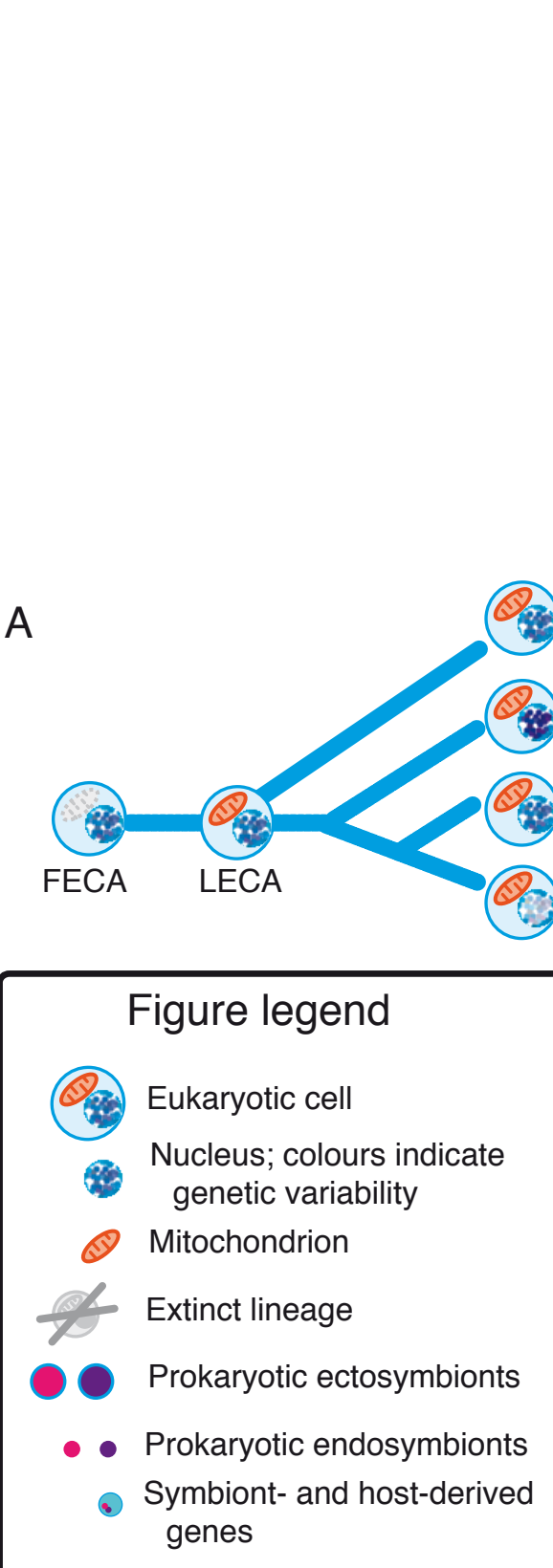

\section{Biological states}

B
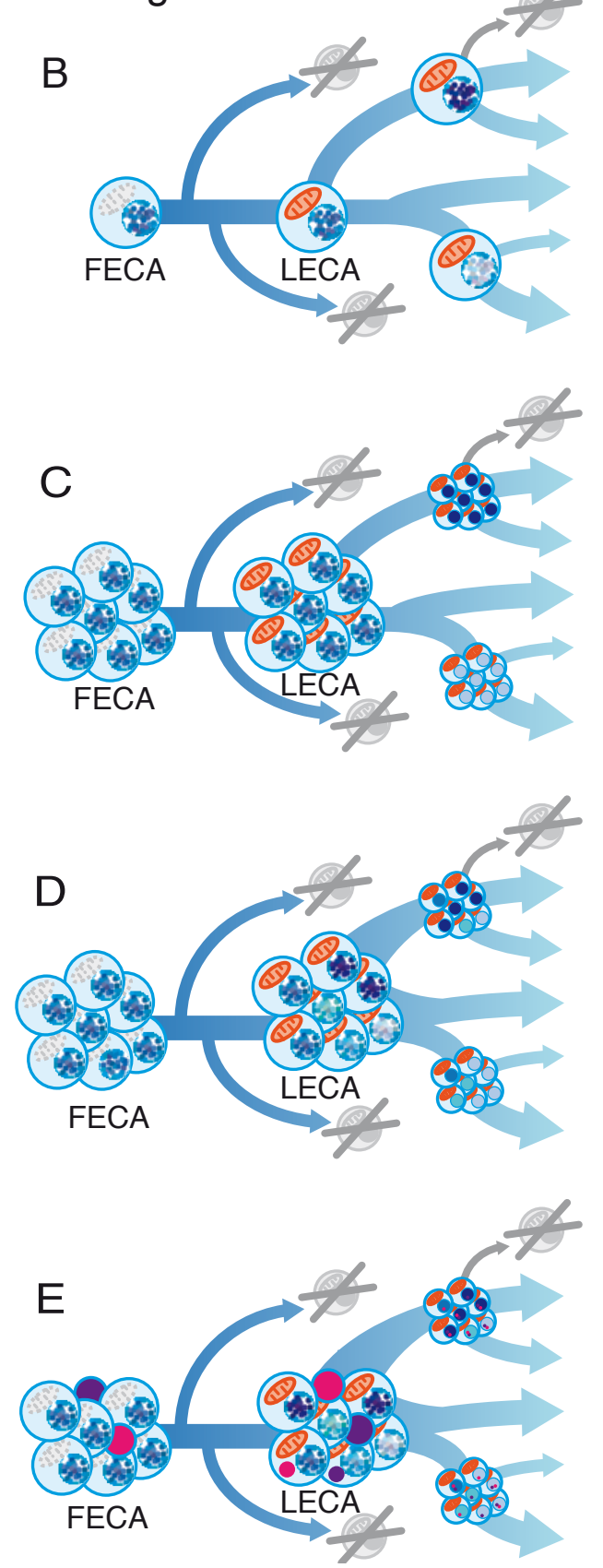

The most common and overarching concept of LECA refers to a phylogenetically inferred ancestral state. This 'state' is abstract: it is a hypothesized ancestral situation that does not refer to a particular biological entity. For example, Mast et al. $\left({ }^{6}\right.$, p. 435$)$ describe LECA as 'a theoretical reconstruction or reference point' (based on a phylogenetic placement), rather than a description of an actual cell or population. Eme et al. $\left({ }^{3}\right.$, p. 714$)$ argue that this 
phylogenetic definition means 'we do not need to know what kind of cellular, genetic and metabolic features [LECA] had in order to be able to identify [its] precise position in the tree of life'.

An important consequence of this view is that knowledge of LECA becomes contingent on current phylogenetic placements, which themselves are based on knowledge of extant eukaryotic diversity. In other words, any attribution of LECA does not refer to a phylogenetic node that is historically fixed. When earlier branching eukaryotes are discovered, the reconstruction referred to as LECA will be replaced by a new one, which will represent an earlier biological state ${ }^{3}$. Or, when a different root of the eukaryotic tree is inferred, this will shift LECA's placement, since it depends on where that root falls ${ }^{7}$.

Beyond any inferred abstract state, what sort of entity was LECA? In other words, how is the biological reality of LECA understood? Even authors advocating a phylogenetic definition of LECA acknowledge that LECA must have had 'organismal' and 'populational' characteristics (e.g., $\left.{ }^{3}\right)$. Most early eukaryote evolution literature refers to LECA as 'a cell' (e.g., ${ }^{7-11}$ ). Only a few papers describe LECA as a population of cells (e.g., ${ }^{6,12,13}$ ). There is even less discussion of whether LECA could have existed as a multispecies consortium, even though consortia figure in several scenarios of eukaryote origins ${ }^{10,14}$.

Altogether, these three possibilities form a continuum of biological LECA concepts. This continuum ranges from a single cell to a population and then to a consortium. All these biological states are then encompassed by the abstract view of LECA as a hypothetical ancestral state (Figure 1). However, none of these views of the potential biological states of LECA is well developed, and yet each has important evolutionary implications. To make unstated assumptions explicit, we now elaborate on each biological concept and their major differences.

\section{A continuum of biological LECA concepts}

The single cell view of LECA sees it as an organism that possessed many widely distributed eukaryotic characters, all of which were encapsulated by a single cell. These features (inferred from phylogenetic reconstructions, as discussed in ${ }^{15}$ ) include mitochondria ${ }^{16}$, a nucleus with genes from at least two origins ${ }^{17}$, the capacity for facultative sex ${ }^{18,19}$, a complex cytoskeleton ${ }^{20}$, and dispersal capabilities in the form of a flagellum ${ }^{20}$ and actin-driven pseudopodia ${ }^{21}$.

This single-cell view of LECA is useful for capturing the cellular and biochemical features that the eukaryote ancestor may have exhibited. However, there may be problems when comparative genomic approaches express LECA claims in reference to single cells. Why? Because genome-based claims about single cells are usually casual shorthand for discussions of ancestral cellular properties that were not necessarily specific to a single cell ${ }^{22-27}$. In other words, genomic inferences track back to populations of genes, rather than any particular single cell (see Box 1 at the end of the text).

When single-cell LECA is posited, it may mean that a genealogical conception of LECA is being imposed on genome data in light of broad knowledge of descent relationships. This genealogical view tracks cell divisions, rather than genomic ancestry, and ultimately arrives at 
a single-cell progenitor of all extant eukaryotes. Although this concept is not clearly articulated in the literature (despite many statements of LECA as a cell), it has been explicitly noted in conceptual discussions of LUCA that distinguish between 'common ancestor' versus 'common ancestry' (e.g., ${ }^{28,29}$; see Box 2 at the end of the text for LECA-LUCA conceptual parallels).

The reasoning in the background of the genealogical view refers to a tree of cells, rather than a tree of genomes (e.g., ${ }^{30}$ ). But importantly, for both the tree of cells and LECA, the only data available for making phylogenetic inferences is genomic. In this crucial sense, therefore, deep phylogenetic reconstruction is about ancestry in the form of a potentially distributed gene pool rather than a specific cellular ancestor ${ }^{28}$. One consequence of pooled ancestry is that individual genes present in any extant population will not all have been inherited from the same ancestral individual (see Box 1). This is the sort of reasoning that informs populational views of ancestry, including LECA. However, there might still be conceptual variability in how populations are understood.

The population view of LECA finds its foundations in standard evolutionary theory that makes populations the basis of evolutionary change. Although variations arise in individual members, evolution relies on the fixation of that variation in reproducing populations ${ }^{31}$. LECA populations are usually conceptualized as a facultatively sexual population of the same cell type with very similar genomes. When conceived in this way (i.e., as the original heterotrophic flagellate population), the population is sometimes argued to have existed over a short timespan in a single area, and to have exploited a limited niche (for discussion, see ${ }^{8}$ ). This view of LECA implies a genomically uniform and geographically localized population that was also probably small in size. However, we do not think that a genetically uniform ancestral state is the only plausible population concept of LECA.

In any population, even if restricted geographically, there will be genetic and phenotypic variation, especially over time. It is perfectly possible (indeed, plausible) that the LECA population existed for numerous generations, during which considerable allelic diversity could have accumulated within the population without causing it to diverge. In such circumstances, LECA could also be conceptualized as a much more heterogeneous population that not only shared a common core of genes, but also had many diverse non-core (accessory) genes. These accessory genes may have allowed subpopulations to inhabit a more diverse, spatially distributed set of niches. When viewed collectively (i.e., all the genes of the subpopulations), these genes would comprise not a single organismal genome but a 'pangenome'. Naturally, there is a continuum that ranges from populations with lower rates of allelic variation to populations with extensive pangenomic variation. Although variation between subpopulations in a pangenomic population can be considerable, various forces (e.g., recombination, migration, niche overlap) can still make the subpopulations one population evolutionarily, rather than genetically separate species.

Pangenomes have been described extensively in prokaryotes, as well as in several eukaryotes (e.g. ${ }^{32-35}$ ). Although the majority of these eukaryotic pangenomes have been identified in plants and fungi, an extant protist lineage, Emiliania huxleyi, has a pangenome that underpins considerable phenotypic plasticity, such that the entity appears in some interpretations as 'a complex of species' ${ }^{33,36}$. If LECA formed a species complex with important phenotypic and genotypic variation, population-LECA could then be conceptually closer to a consortium than a still-cohesive population. 
The consortium view of $L E C A$, as an association of cells from different lineages rather than a single cell or even a single population, lies at the far end of our conceptual continuum. Although consortia are mentioned in the literature in relation to eukaryogenesis (e.g., ${ }^{10,37}$ ), this concept is not applied once LECA is achieved. The reason for this conceptual absence is that LECA is accepted as a fully cellular entity, the components of which are reliably coinherited. Any consortium-oriented views of early eukaryotic evolution are always about early eukaryotic or protoeukaryotic lineages in the transitional period between FECA and LECA. Several articulations of the transitional period after FECA explicitly invoke consortia scenarios about different genetic lineages in the process of fusing into one (e.g., ${ }^{10,14,38-40}$ ). For example, a recent consortium formulation describes the extended transition period from FECA to LECA as consisting of 'serial symbiotic associations with different partners ... the existence of prokaryotic consortia or gradual waves of HGT' ${ }^{41}$.

Carl Woese's ${ }^{37,42}$ progenote concept of the pre-LUCA state is relevant here (see Box 2). He postulated the mixing and eventual 'annealing' of different genetic resources, which eventually produced new units of selection that we now consider the most ancestral forms of life. Once this new unit is the focus of selection (rather than each separate but interacting lineage), a major transition in evolution has occurred ${ }^{43}$. Thinking about this concept of LUCA helps explain why consortia scenarios are not really viable for LECA concepts. LECA is already a new unit of selection and no longer a separable collection of lineages that can still be called a consortium.

However, there is at least one way in which LECA could be conceptualized as a consortium, and which has intriguing evolutionary and phylogenetic implications. This scenario is based on a loose association between a eukaryotic host and multiple prokaryotic symbionts. Their relationship would be analogous to the endosymbionts of modern amoebae ${ }^{44}$ or to the ectosymbionts of oxymonads ${ }^{45}$. A LECA consortium of this sort might have given rise to daughter associations that differentially lost symbionts. Such losses would eventually lead to a situation in which all extant eukaryotes lack these symbionts, but different groups of eukaryotes would have different lateral gene transfer (LGT) signatures from ancient cryptic symbiotic events. Although consortium views such as this do not yet have evidential support, they could help explain some messy phylogenetic signal that obscures efforts to reconstruct eukaryote evolutionary history.

Overall, our analysis of the three biological positions on the continuum (single cell, population, consortium) shows that populations are the evolutionary concept most realistically underpinning the comparative genomic reconstructions focused on LECA. This is the case even when a genealogical view is superimposed on genomic inferences. Furthermore, there are good reasons to consider a pangenomic LECA to be as conceptually viable as a genetically homogenous LECA. Given the timescale over which LECA is likely to have existed, and the potential of any population for variation, we suggest that conceptualizing LECA as a pangenomic population is theoretically promising, despite its absence from the literature. This novel concept warrants explicit discussion because it has important evolutionary ramifications that can inform specific hypotheses about early eukaryotic evolution. 


\section{Evolutionary aspects of pangenomic LECA}

All evolving populations possess the potential for diversification, and that potential is actualized by geographical and ecological conditions that lead to fitness losses and gains. LECA was no different. By analysing the implications of a pangenomic population structure for LECA, we can further reason about the ancestral environment in relation to the cellular features exhibited by populations of LECA cells. Doing so may enhance our evolutionary insights into LECA.

\section{Geographical and ecological scenarios}

Should we restrict LECA to one particular kind of environment? A pangenomic view of LECA might also mean that LECA was distributed across a wider range of environments, with more diverse niche-specific genes. Would LECA have had a small or large population size? Individual strains with particular accessory genes may have been small, localized populations, rarely interacting ecologically with the other strains. If accessory genes are niche-defining, then imagining a single environment for LECA is problematic. In other words, expecting to find very specific environmental conditions that determine exactly what LECA was and could do may not be appropriate if a pangenomic entity lies at the base of the eukaryotic tree.

Fitness and adaptiveness of LECA

It is commonly assumed that pangenomes, whether in prokaryotes or eukaryotes, correlate in size with capacities for the organismal group to have plastic phenotypes and spread widely (e.g., ${ }^{33,46,47}$ ). These observations lead to suggestions that having a pangenome is adaptive for members of the pangenomic population ${ }^{48}$, which could imply that the larger the pangenome the better. Any long-term persistence of genetic differences between strains suggests a fitness benefit for those differences. Is this how we should think about LECA?

Prokaryotic pangenomics is divided on this question of the adaptiveness of pangenomes. One argument that has been made for adaptiveness is that pangenomes are common and permit environmental flexibility, and so must have been selected for ${ }^{48}$. Moreover, continues this argument from a population-genetic point of view, because large populations are more likely to have large pangenomes, neutral or deleterious additions to pangenomes will not persist under the higher selection efficiency experienced by these large prokaryotic populations ${ }^{48,49}$. However, population-genetic reasoning has also been invoked to support the opposite argument: that even though large effective population sizes correlate with large pangenomes, because the majority of genetic differences are necessarily selectively neutral (or nearly so), pangenomes will mostly be explained by drift and other non-adaptive processes ${ }^{50,51}$ (see ${ }^{52}$ for a cautionary note about applying population genetic theory to pangenomes).

Whichever way this debate is ultimately reconciled, it is clearly the case that population size matters for how we conceptualize LECA. Although defining effective population size is problematic for prokaryotic and eukaryotic microorganisms ${ }^{53,54}$, there are established lines of reasoning that can be applied to LECA. If either LECA or its subpopulations had small effective population sizes, this would mean that the population(s) probably carried more deleterious mutations (including deleterious laterally transferred genes) or at least more unpurged variation. Small population sizes mean that more mutations are tolerated because selection is not so effective. Given that LECA persisted and diversified successfully, it might make more sense to assume LECA had a large effective population size, with adaptive accessory genes in the smaller subpopulations comprising the meta-population. Some of 
those smaller subpopulations may have been burdened with a greater abundance of deleterious mutations, in which case extinction scenarios are very likely, and would in fact be expected in a period of rapid diversification.

\section{The diversification of LECA}

A major implication of a pangenomic LECA population would be its impact on our understanding of the diversification of eukaryotes. If LECA were a complex, genetically and phenotypically variable population exploring phylogenetic and ecological space, then the rapid diversification of eukaryotes might be more easily achieved. In other words, a pangenomic view is one in which multilineage speciation is more likely than not. Although details of the diversification of extant eukaryotes are still obscure ${ }^{23}$, both clumped and staggered speciation rates are compatible with the notion of LECA as a pangenomic entity.

\section{LECA capacities and functions}

It is common now to discuss LECA as a complex cell, with a large gene repertoire and many of the characteristics we understand as core features of extant eukaryotes (e.g., ${ }^{7,55}$ ). However, if we take a pangenomic view of LECA, the complexity of any single cell in the group may be undermined, due to the whole range of LECA's genetic resources being distributed across numerous strains and not necessarily found in every single cell (even if genealogically, specific genes can be traced back to particular cellular ancestors - see Box 1). A sufficiently 'open' pangenome might mean that each strain held a number of genes not shared with other strains. Those distributed genes might now be found patchily in extant eukaryotes (Fig. 2). This view does not question that LECA possessed eukaryote-defining structures and capacities at the phenotypic level (e.g., nucleus, mitochondria), but is concerned with the variability of the genes contributing to these and other traits. In other words, reconstruction of traits that were present in 'the last eukaryotic common ancestor' is better understood as a summary of resources in an ancestral population in which variation was more likely than not.

\section{Figure 2. Explaining trait distributions in extant eukaryotes}

Two different explanations for trait distributions in extant lineages of eukaryotes (from an imagined viewpoint of total knowledge) are depicted. Cells at the top represent extant supergroups (e.g., Opisthokonta, SAR, Excavata). Extinct lineages are faded out with grey crosses.

\section{A. LECA as a homogeneous population.}

The actual ancestral state of LECA is represented as ABCDEF. However, from the genomes of extant lineages it is inferred to be only $A B$. The differences between extant populations are explained by gene gains and losses in the evolution of each lineage. $C, D$, and $F$ are thus inferred as post-LECA innovations or acquisitions.

B. LECA as a heterogeneous population.

Cells within the LECA population (within the grey oval) represent LECA subpopulations with different genomic compositions. Dotted lines indicate gene flow between subpopulations. The ancestral reconstructed state of LECA is distributed across a heterogeneous population such that no individual subpopulation (individual cells) within the greater population contains the entire LECA genome (ABCDEFG). Different subpopulations possess the core $(A B)$ genes but also have a variety of accessory genes (C, D, E, and F). Retention of these accessory genes in extant eukaryotic lineages could be explained by their ancestry in particular subpopulations 
of LECA. For example, $\mathrm{C}$ is present in two distantly related extant eukaryotic lineages not because of descent from a single ancestor containing $C$, but because those lineages inherited $C$ from two LECA subpopulations that independently possessed it. To wit, they were not part of directly interbreeding subpopulations, nor did they share a common ancestral subpopulation that contained $\mathrm{C}$. They independently acquired $\mathrm{C}$ by interbreeding with other subpopulations. This process is similar to how genes might be passed from one subpopulation to a distant subpopulation in a ring species.

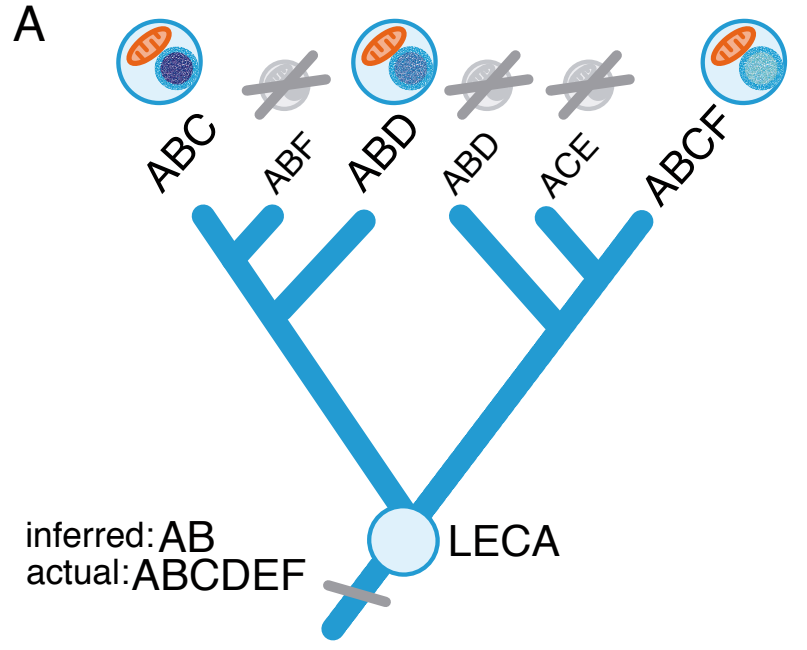

Homogeneous LECA

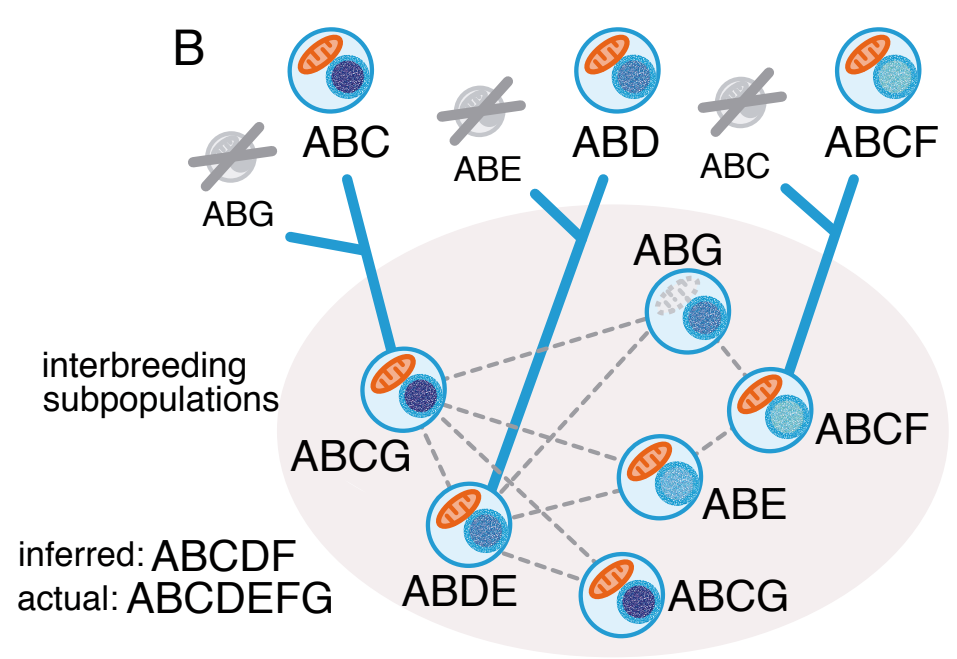

Heterogeneous LECA

One objection to this reasoning is that pangenomic LECA could be imagined as a descendant of a more homogeneous LECA population that only later diversified. If that were the case, the 'true' LECA would be the homogeneous (non-pangenomic) ancestral population. However, we are suggesting that there may not be an evolutionarily significant period of homogeneity for LECA. The innovations that arose with LECA gave this population a capacity to explore and create new niches; these novel features would have undergone a range of different evolutionary pressures ${ }^{8}$. In such a situation, genetic fluidity would be a better description of LECA than stasis. Biologically and evolutionarily, as long as all the capacities attributed to LECA arose within and were distributed differentially across subpopulations, and as long as enough gene flow occurred between these LECA subpopulations, then the pangenomic population counts as LECA.

This pangenomic view might then affect ancestral reconstructions of characteristics of LECA (e.g. ${ }^{2,56}$ ) or its subcellular compartments ${ }^{57}$. If it is unclear whether any given gene was present in the LECA core genome, or whether it was an accessory gene in the LECA pangenome, then it becomes even less clear how to reconstruct pathways present in any given single cell within LECA (see Fig. 2). Different enzymes or even whole biochemical pathways might have been distributed across different subpopulations. Accordingly, some structures or biochemical pathways 'present in LECA' might not all have existed intact and operational in every - or even any - subpopulation of cells. To some extent, similar uncertainty exists even when LECA is conceived as a single cell: mere spatial coexistence within a single (part of a) cell is no guarantee of temporal coexistence, let alone cellular or 
biochemical interaction. However, the difficulties in assigning multiple ancestral genes to a single cell within a pangenomic LECA population would increase the uncertainty surrounding functional inferences.

A genealogical perspective might say that LECA only comes into existence when all functional capacities operate in each cell of the population, and that the population descends from a single ancestral cell with all those structural and functional features. However, claims like this are too strong given data sources (genomic, and thus not allowing functional certainty) and too idealized in an evolutionary sense (not taking populational variation into account, nor the need for LECA traits to be fixed in a population).

What happens if there are different evolutionary explanations for different parts of any pangenome? Conceptualizing LECA pangenomically also affects interpretations of patchily distributed genes or characters (Figure 2). The likelihood of gene or character gains, convergence, losses, or LGTs might be different depending on how we understand LECA's genome. In a pangenomic scenario, a gene present in the ancestral accessory genome might be retained in the accessory genome of many daughter lineages, but lost from others at a higher rate than core genes. This occurs in prokaryotes, where it has been shown that the core genome is under greater selective constraints than the pangenome as a whole ${ }^{58,59}$ (for nuances, see ${ }^{60}$ ), and is less prone to LGT ${ }^{61}$. Eukaryotic pangenome dynamics and their mechanisms are poorly understood, particularly because of sexual reproduction. However, similar dynamics to prokaryotes may have occurred in pangenomic LECA, which would affect and potentially explain gene distributions in their descendants.

Conversely, accessory genes are known in many cases to be more prone to gene deletion, even in eukaryotic pangenomes ${ }^{35}$. If LECA were an already complex entity, and the simplification of complexity allowed for advantageous innovations ${ }^{7}$, then a capacity for gene deletion could help explain the evolvability of LECA into many successful eukaryotic lineages.

How can it be decided which genes were core in LECA and which were not? A patchily distributed gene present in some eukaryotic groups but not others can be explained either by LGT or multiple losses. In a pangenomic view of LECA, particular patchily distributed genes could be explained by the existence of an ancestral pangenome (Fig. 2). However, it is unclear which patchily distributed genes are best explained by LGT and/or multiple losses, or by pangenomic ancestry. There is already considerable debate about the existence of pangenomes in extant eukaryotes, and the extent of gene transfer in eukaryotic evolutionary history ${ }^{62-64}$, even though mechanisms other than LGT can lead to pangenomes in eukaryotes $\left(\right.$ see $\left.^{33,34,36}\right)$. Can a pangenomic view of LECA contribute further insight to this debate?

\section{Phylogenetic implications}

The pangenomic nature of alphaproteobacterial and cyanobacterial lineages has been advanced as an explanation for the difficulties in identifying clear prokaryotic sister lineages to genes of organellar origin (e.g., ${ }^{7,65,66}$ ). Taking a pangenomic view of LECA might therefore influence how we should think of the bases of later eukaryote groups. Evidence for pangenomic eukaryotes has only come to light within the last few years due to more extensive sequencing projects, and so it is possible that with more such work, we will find eukaryotic pangenomes to be more widespread than expected. However, even in prokaryotes, where more is known, not all prokaryotic populations have pangenomic structure. In addition, the extent of genetic variation in prokaryotic and eukaryotic 
pangenomes can vary considerably (see ${ }^{48}$, Table 1 ). Despite these uncertainties, a broadly pangenomic concept of LECA would raise important general questions such as whether a pangenomic scenario can be ruled out for any ancestral branching point, when exactly genomic variability might have decreased in some lineages, and why.

Can mixed phylogenetic signal and the ongoing difficulties involved in pinpointing the root of the eukaryotic tree be explained by an already diverse LECA (Fig. 2)? It is certainly possible that a genetically variable LECA might affect deep phylogenomic analyses, and that examples of apparent 'mixed signal' are real. Currently, however, a more probable explanation (or at least a more conservative one) would be phylogenetic artefacts resulting from poor taxon sampling for 'deep-branching' eukaryotes, and from oversimplifications inherent in existing phylogenetic models ${ }^{23}$. These artefacts then combine with the loss of phylogenetic signal that is due to the time elapsed since the clades diverged. Nevertheless, thinking about a pangenomic LECA can still flesh out biologically and constrain what is said evolutionarily about the abstract phylogenetic state that reconstructions produce. Different concepts of LECA may thus indicate where greater methodological subtleties are required in order to capture this ancestral reality.

\section{Concluding perspectives}

Our analysis shows that by probing the conceptual machinery used in any LECA investigation (under any particular hypothesis of the evolutionary origin of eukaryotes), reasoning can be tightened and the evolutionary implications of different concepts made more transparent. Doing so may help evaluate competing hypotheses. In particular, we suggest that taking the possibility of pangenomic LECA seriously will allow fuller evolutionary reasoning about the phylogenetic inferences made from genomic data.

Our core message is that viewing LECA as a population cluster with a variable genomic resource (i.e., pangenomically), plus an extended geographic and temporal presence, is not only evolutionarily plausible but also biologically credible. However, LECA is seldom if ever viewed in this way, and most conceptions instead rely on a narrowly fixed genetic and phenotypic state.

Current knowledge does not allow us to decide between LECA as a genetically homogeneous or a pangenomically variable population structure. This equipossibility means it would be prudent to consider both states when discussing the nature of LECA. We also show that although it can be convenient to talk about LECA as a single cell, this may mislead researchers who interpret shorthand expressions at face value. Genealogical conceptions of LECA that trace back to a single cell should not be conflated with tracing genomic ancestry, for which the source will be a population. Taking a more complex view of LECA, such as a population with pangenomic structure, will complicate already demanding considerations in the phylogeny of eukaryotes, but may also inform more sophisticated reconstructive efforts. At the very least, broader concepts of LECA - including the novel pangenomic view we outline above - will influence how those reconstructions are interpreted biologically, ecologically, and evolutionarily. 


\section{Box 1: Genealogy of common ancestor versus ancestral state}

Imagine an attempt to reconstruct the gene content of another last common ancestor of a number of sexually reproducing lineages: the last common ancestor of primates (LCAP). As with efforts to reconstruct LECA's gene content, reconstructing LCAP would involve surveying the genomes of extant primates, then determining the likelihood of any given shared gene having been vertically inherited from the last common ancestor, or instead having arisen in a later lineage.

What is being reconstructed in this case? All primates can certainly trace their lineage back to an asexually reproducing organism (analogous to the clonal reproduction that gives rise to the concept of the 'tree of cells'). However, this asexual ancestor lived long before the emergence of primates, and is assuredly not the target of LCAP reconstructions. All primates can also trace their lineage to a single individual from which they are each descended matrilineally. This ancestor contributed each primate's mitochondria (the mitochondrial Eve). However, the 'mitochondrial Eve' of primates is not the only common ancestor of all living primates: She is only the easiest common ancestor to infer, because of the lack of recombination of mitochondrial DNA. Indeed, any segment of DNA that has not undergone homologous recombination can be traced back to a different shared common ancestor.

Because these other common ancestors are different individuals, none of them - not even the one that lived most recently - correspond to a single individual whose gene content is inferred from reconstructions of LCAP. Instead, these reconstructions produce a probabilistic snapshot of gene content across the entire population. Although in a genetically homogeneous population, the genealogical last common ancestor will have high probabilities of having the same gene content as this probabilistic reconstruction, this is not necessarily the case in a genetically variable population. The genetically variable case is what we are exploring in our analysis of pangenomic LECA. 


\section{BOX 2: A conceptual parallel? Woese's concept of LUCA}

A brief overview of the concept of LUCA (the Last Universal Common Ancestor) is instructive for understanding how concepts influence evolutionary hypotheses. In the LUCA literature, there is a strong tradition of discussing the origin of life as a consortium composed of different lineages of genetic material (e.g., ${ }^{67-69}$; see ${ }^{70}$ for a countervailing view). This possibility has become dominant in the field due to a scenario sketched out by Carl Woese ${ }^{37,42,71}$. Woese argued explicitly that LUCA should be conceived as a multilineage consortium, even if that association were not composed of organismal lineages with genetic fidelity.

The 'progenote' was Woese's name for this association of loosely differentiated protocellular entities ${ }^{37,72}$. He postulated this scenario in order to understand how lateral gene transfer (LGT) may have operated early in the origin of life, and how once stricter cellular separation of lineages began, the consortium stepped over the 'Darwinian threshold'. Evolution as we understand it - based on the distinct heritability of genotypes and phenotypes - began with the origin of true cellular lineages. Selection began to operate on specific populations, rather than the processes occurring within the genetically promiscuous progenote association.

Woese's argument has had a strong impact on anyone discussing LUCA, on the one hand for its emphasis on the evolutionary importance of LGT and on the other with regard to how LUCA is conceptualized. As Doolittle explains ${ }^{73}$, the progenote's genetic heterogeneity effectively ruled out origin scenarios 'of a single first kind of cell as the progenitor of all contemporary life'. Ancestry, in other words, should not be conflated with an ancestor ${ }^{28,29}$. In addition, Woese's conceptualization also raised fundamental questions about whether phylogenetic methods could ever untangle these tangled evolutionary origins.

Woese then went on to suggest that the ancestral states of the three domains, including eukaryotes and thus potentially LECA, was 'to some extent communal, but in a much more local, restricted sense than that which holds for the universal ancestor [LUCA]' ${ }^{37}$. If this is correct, he continued, 'the major lineages within each organismal domain would not sort out cleanly along phylogenetic lines: there would be many conflicting gene histories for the deep branchings within each domain.'

Nowadays, most early eukaryote evolutionary biologists would interpret Woese to be referring to a lengthy transitional state preceding LECA, not LECA itself, when he mused about a consortium lying at the base of the eukaryote tree. Nevertheless, his earlier progenote concept demonstrates clearly how conceptualization matters for phylogenetic reconstruction and evolutionary hypotheses. This is the motivating idea for our analysis of LECA concepts. 


\section{Acknowledgements}

We gratefully acknowledge Sergio Muñoz-Gómez for several thoughtful discussions and the inspiration for Figure 2. We also thank Ford Doolittle and Andrew Roger for insightful comments on earlier drafts of this paper. For views on the conceptual continuum of LECA, we are grateful to anonymous attendees at the 2017 EMBO workshop on Comparative Genomics of Eukaryotic Microbes, and members of the Dalhousie University Centre for Comparative Genomics and Evolutionary Bioinformatics. MAO's research is supported by the French government via the 'Investments for the future' Programme, IdEx Bordeaux (ANR-10-IDEX03-02). MML is supported by a Marie Skłodowska-Curie Individual Fellowship under the EU

Framework Programme for Research and Innovation Horizon 2020 (Project ID 747789). JGW was supported by a College for Life Sciences Fellowship at the Wissenschaftskolleg zu Berlin. IR-T was supported by a European Research Council Consolidator Grant (ERC-2012Co -616960) grant, and funding (BFU2017-90114-P) from Ministerio de Economía y Competitividad (MINECO), Agencia Estatal de Investigación (AEI), and Fondo Europeo de Desarrollo Regional (FEDER).

\section{References}

1 Roger, A. J., Muñoz-Gomez, S. A. \& Kamikawa, R. Curr Biol 27, R1177-R1192, doi:10.1016/j.cub.2017.09.015 (2017).

2 Makarova, K. S., Wolf, Y. I., Mekhedov, S. L., Mirkin, B. G. \& Koonin, E. V. Nucleic Acids Res 33, 4626-4638, doi:10.1093/nar/gki775 (2005).

3 Eme, L., Spang, A., Lombard, J., Stairs, C. W. \& Ettema, T. J. G. Nat Rev Microbiol 15, 711-723, doi:10.1038/nrmicro.2017.133 (2017).

4 Zaremba-Niedzwiedzka, K. et al. Nature 541, 353-358, doi:10.1038/nature21031 (2017).

5 Field, M. C. \& Dacks, J. B. Curr Opin Cell Biol 21, 4-13, doi:10.1016/j.ceb.2008.12.004 (2009).

6 Mast, F. D., Barlow, L. D., Rachubinski, R. A. \& Dacks, J. B. Trends Cell Biol 24, 435442, doi:10.1016/j.tcb.2014.02.003 (2014).

7 Koonin, E. V. Genome Biol 11, 209, doi:10.1186/gb-2010-11-5-209 (2010).

$8 \quad$ Butterfield, N. J. Front Palaeontol 58, 5-17 (2015).

9 Poole, A. M. \& Neumann, N. Res Microbiol 162, 71-76, doi:10.1016/j.resmic.2010.10.002 (2011).

10 Margulis, L., Chapman, M., Guerrero, R. \& Hall, J. Proc Natl Acad Sci U S A 103, 13080-13085, doi:10.1073/pnas.0604985103 (2006).

11 Klöpper, T. H., Kienle, N., Fasshauer, D. \& Munro, S. BMC Biol 10, 71, doi:10.1186/1741-7007-10-71 (2012).

12 Vaškovičová, K. et al. Biol Direct 8, 8, doi:10.1186/1745-6150-8-8 (2013).

13 Lane, N. Biol Direct 6, 35, doi:10.1186/1745-6150-6-35 (2011).

14 Moreira, D. \& López-Garcia, P. J Mol Evol 47, 517-530 (1998).

15 Koumandou, V. L. et al. Crit Rev Biochem Mol Biol 48, 373-396, doi:10.3109/10409238.2013.821444 (2013).

16 Hampl, V. et al. PLoS One 3, e1383, doi:10.1371/journal.pone.0001383 (2008).

17 Rivera, M. C., Jain, R., Moore, J. E. \& Lake, J. A. Proc Natl Acad Sci U S A 95, 62396244 (1998).

18 Dacks, J. \& Roger, A. J. J Mol Evol 48, 779-783 (1999). 
Speijer, D., Lukeš, J. \& Eliaš, M. Proc Natl Acad Sci U S A 112, 8827-8834, doi:10.1073/pnas.1501725112 (2015).

20

Yubuki, N. \& Leander, B. S. Plant J 75, 230-244, doi:10.1111/tpj.12145 (2013).

21 Richards, T. A. \& Cavalier-Smith, T. Nature 436, 1113-1118, doi:10.1038/nature03949 (2005).

22 Aravind, L., Burroughs, A. M., Zhang, D. \& lyer, L. M. Cold Spring Harb Perspect Biol 6, a016063, doi:10.1101/cshperspect.a016063 (2014).

23 Eme, L., Sharpe, S. C., Brown, M. W. \& Roger, A. J. Cold Spring Harb Perspect Biol 6, doi:10.1101/cshperspect.a016139 (2014).

24 Poole, A. M. \& Gribaldo, S. Cold Spring Harb Perspect Biol 6, a015990, doi:10.1101/cshperspect.a015990 (2014).

25 van Hooff, J. J., Tromer, E., van Wijk, L. M., Snel, B. \& Kops, G. J. EMBO Rep 18, 1559-1571, doi:10.15252/embr.201744102 (2017).

López-Garcia, P. \& Moreira, D. Trends Ecol Evol 30, 697-708, doi:10.1016/j.tree.2015.09.005 (2015).

27

He, D. et al. Curr Biol 24, 465-470, doi:10.1016/j.cub.2014.01.036 (2014).

28 Doolittle, W. F. Philos Trans R Soc Lond B Biol Sci 364, 2221-2228, doi:10.1098/rstb.2009.0032 (2009).

29

30

Vetsigian, K., Woese, C. \& Goldenfeld, N. Proc Natl Acad Sci U S A 103, 10696-

10701, doi:10.1073/pnas.0603780103 (2006).

Lerat, E., Daubin, V. \& Moran, N. A. PLoS Biol 1, E19, doi:10.1371/journal.pbio.0000019 (2003).

31

32

33

34

35

Lewontin, R. C. Annu Rev Ecol Evol Syst 1, 1 - 18 (1970).

Song, G. et al. PLoS One 10, e0120671, doi:10.1371/journal.pone.0120671 (2015).

Read, B. A. et al. Nature 499, 209-213, doi:10.1038/nature12221 (2013).

Golicz, A. A., Batley, J. \& Edwards, D. Plant Biotechnol J 14, 1099-1105,

doi:10.1111/pbi.12499 (2016).

Plissonneau, C., Hartmann, F. E. \& Croll, D. BMC Biol 16, 5, doi:10.1186/s12915-0170457-4 (2018).

Bendif, E. et al. Front Microbiol 7, 784, doi:10.3389/fmicb.2016.00784 (2016).

Woese, C. Proc Natl Acad Sci U S A 95, 6854-6859 (1998).

Lopez-Garcia, P. \& Moreira, D. Trends Biochem Sci 24, 88-93 (1999).

39

40

Bell, P. J. Ann N Y Acad Sci 1178, 91-105, doi:10.1111/j.1749-6632.2009.04994.x (2009).

Booth, A. \& Doolittle, W. F. Proc Natl Acad Sci U S A 112, 10278-10285,

doi:10.1073/pnas.1421376112 (2015).

41 Pittis, A. A. \& Gabaldón, T. Nature 531, 101-104, doi:10.1038/nature16941 (2016).

42 Woese, C. R. Microbiol Rev 51, 221-271 (1987).

43 Szathmáry, E. \& Smith, J. M. Nature 374, 227-232, doi:10.1038/374227a0 (1995).

44

45 Horn, M. \& Wagner, M. J Eukaryot Microbiol 51, 509-514 (2004).

lida, T., Ohkuma, M., Ohtoko, K. \& Kudo, T. FEMS Microbiol Ecol 34, 17-26 (2000). Brito, P. H. et al. Genome Biol Evol 10, 108-124, doi:10.1093/gbe/evx270 (2018). von Dassow, P. et al. ISME J 9, 1365-1377, doi:10.1038/ismej.2014.221 (2015).

48 Mclnerney, J. O., McNally, A. \& O'Connell, M. J. Nat Microbiol 2, 17040, doi:10.1038/nmicrobiol.2017.40 (2017).

49 Mclnerney, J., Pisani, D. \& O'Connell, M. J. Philos Trans R Soc Lond B Biol Sci 370, 20140323, doi:10.1098/rstb.2014.0323 (2015).

50 Andreani, N. A., Hesse, E. \& Vos, M. ISME J 11, 1719-1721, doi:10.1038/ismej.2017.36 (2017). 
Vos, M. \& Eyre-Walker, A. Nat Microbiol 2, 1576, doi:10.1038/s41564-017-0067-5 (2017).

52 Shapiro, B. J. Nat Microbio/ 2, 1574, doi:10.1038/s41564-017-0066-6 (2017).

53 Rocha, E. P. C. Mol Biol Evol 35, 1338-1347, doi:10.1093/molbev/msy078 (2018).

54 Sung, W., Ackerman, M. S., Miller, S. F., Doak, T. G. \& Lynch, M. Proc Natl Acad Sci U $S$ A 109, 18488-18492, doi:10.1073/pnas.1216223109 (2012).

55 Lang, B. F. \& Burger, G. Adv Bot Res 63, 1-20 (2012).

56 Zmasek, C. M. \& Godzik, A. Genome Biol 12, R4, doi:10.1186/gb-2011-12-1-r4 (2011).

57 Gabaldón, T. \& Huynen, M. A. Science 301, 609, doi:10.1126/science.1085463 (2003).

58 Sun, S., Xiao, J., Zhang, H. \& Zhang, Z. Front Microbiol 7, 1180, doi:10.3389/fmicb.2016.01180 (2016).

59 Bohlin, J., Eldholm, V., Pettersson, J. H., Brynildsrud, O. \& Snipen, L. BMC Genomics 18, 151, doi:10.1186/s12864-017-3543-7 (2017).

60 Castillo-Ramirez, S. et al. PLoS Pathog 7, e1002129, doi:10.1371/journal.ppat.1002129 (2011).

61 Gordienko, E. N., Kazanov, M. D. \& Gelfand, M. S. J Bacteriol 195, 2786-2792, doi:10.1128/JB.02285-12 (2013).

62 Boto, L. Bioessays 40, doi:10.1002/bies.201800001 (2018).

63 Leger, M. M., Eme, L., Stairs, C. W. \& Roger, A. J. Bioessays 40, e1700242, doi:10.1002/bies.201700242 (2018).

64 Martin, W. F. Bioessays 39, doi:10.1002/bies.201700115 (2017).

65 Thiergart, T., Landan, G., Schenk, M., Dagan, T. \& Martin, W. F. Genome Biol Evol 4, 466-485, doi:10.1093/gbe/evs018 (2012).

$66 \mathrm{Ku}, \mathrm{C}$. et al. Proc Natl Acad Sci U S A 112, 10139-10146, doi:10.1073/pnas.1421385112 (2015).

67 Koonin, E. V. Nat Rev Microbiol 1, 127-136, doi:10.1038/nrmicro751 (2003).

68 Mushegian, A. Front Biosci 13, 4657-4666 (2008).

69 Glansdorff, N., Xu, Y. \& Labedan, B. Biol Direct 3, 29, doi:10.1186/1745-6150-3-29 (2008).

70 Forterre, P. Res Microbiol 159, 74-80, doi:10.1016/j.resmic.2007.12.001 (2008).

71 Woese, C. R. Proc Natl Acad Sci U S A 99, 8742-8747, doi:10.1073/pnas.132266999 (2002).

72 Gogarten, J. P. \& Olendzenski, L. Curr Opin Genet Dev 9, 630-636 (1999).

73 Doolittle, W. F. Sci Am 282, 90-95 (2000). 